\title{
Effect of training in hypoxia on repeated sprint performance in female athletes
}

Nobukazu Kasai', Sahiro Mizuno', Sayuri Ishimoto², Etsuko Sakamoto², Misato Maruta² and Kazushige Goto ${ }^{1,2^{*}}$

\begin{abstract}
Background: This study determined the effect of repeated sprint training in hypoxia (RSH) in female athletes.

Methods: Thirty-two college female athletes performed repeated cycling sprints of two sets of $10 \times 7$-s sprints with a 30-s rest between sprints twice per week for 4 weeks under either normoxic conditions (RSN group; $\mathrm{F}_{i} \mathrm{O}_{2}, 20.9 \%$; $\mathrm{n}=16$ ) or hypoxic conditions (RSH group; $\left.\mathrm{F}_{i} \mathrm{O}_{2}, 14.5 \% ; \mathrm{n}=16\right)$. The repeated sprint ability $(10 \times 7$-s sprints) and maximal oxygen uptake $\left(\dot{\mathrm{V}}_{2} \mathrm{max}\right)$ were determined before and after the training period.

Results: After training, when compared to pre-values, the mean power output was higher in all sprints during the repeated sprint test in the RSH group but only for the second half of the sprints in the RSN group $(P \leq 0.05)$. The percentage increases in peak and mean power output between before and after the training period were significantly greater in the RSH group than in the RSN group (peak power output, $5.0 \pm 0.7 \%$ vs. $1.5 \pm 0.9 \%$, respectively; mean power output, $9.7 \pm 0.9 \%$ vs. $6.0 \pm 0.8 \%$, respectively; $P<0.05)$. $\dot{\mathrm{V}} \mathrm{O}_{2 \mathrm{max}}$ did not change significantly after the training period in either group.
\end{abstract}

Conclusion: Four weeks of RSH further enhanced the peak and mean power output during repeated sprint test compared with RSN.

Keywords: Normobaric hypoxia, Repeated sprint ability, Anaerobic power output, Team sport athletes

\section{Background}

The application of training in hypoxia for exercise capacity improvement has been accepted widely among endurance athletes (e.g., long-distance runners and swimmers), and a large amount of experimental evidence supports the efficacy of this training method (Dufour et al. 2006; Millet et al. 2010; Strzala et al. 2011). In contrast, growing evidence suggests that training in hypoxia also improves anaerobic or repeated sprints performance (Mizuno et al. 1990; Hamlin et al. 2010; Faiss et al. 2013a, 2015; Gatterer et al. 2014). Mizuno et al. (1990) revealed that 2 weeks of training at altitude significantly increased the time to exhaustion (TTE) in highly trained cross-country skiers during incremental treadmill running, with no change in maximal oxygen uptake $\left(\dot{\mathrm{V}} \mathrm{O}_{2 \mathrm{max}}\right)$. Furthermore, improvements in running duration following

\footnotetext{
*Correspondence: kagoto@fc.ritsumei.ac.jp

${ }^{2}$ Faculty of Sport and Health Science, Ritsumeikan University, 1-1-1

Nojihigashi, Kusatsu, Shiga 525-8577, Japan

Full list of author information is available at the end of the article
}

the training period were significantly correlated with an increase in the buffering capacity of the gastrocnemius muscle. Moreover, intensive training in hypoxia $\left(\mathrm{F}_{\mathrm{i}} \mathrm{O}_{2}\right.$, $17.0-14.0 \%)$ for 10 successive days caused significantly greater increases in the mean power output during $30 \mathrm{~s}$ of maximal pedaling than did the same training in normoxia (Hamlin et al. 2010). Therefore, sprint training in hypoxia may further improve the anaerobic power output and sprint capacity (Billaut et al. 2012).

Team sports such as football, hockey, and basketball require athletes to perform a number of short sprints separated by periods of rest or low-to-moderate-intensity exercise (Bishop and Edge 2006). Recent studies have focused on the influences of repeated sprint training in hypoxia (RSH) on repeated sprint ability (Faiss et al. 2013a; Galvin et al. 2013; Millet et al. 2013; Gatterer et al. 2014). Faiss et al. (2013a) showed that 4 weeks of RSH further increased the number of sets until fatigue during a repeated sprint test (repeated 10-s maximal sprints with 20-s active recovery until exhaustion) compared with

\section{Springer}

(c) 2015 Kasai et al.. This article is distributed under the terms of the Creative Commons Attribution 4.0 International License (http://creativecommons.org/licenses/by/4.0/), which permits unrestricted use, distribution, and reproduction in any medium, provided you give appropriate credit to the original author(s) and the source, provide a link to the Creative Commons license, and indicate if changes were made. 
repeated sprint training in normoxia (RSN) among male cyclists. Furthermore, Gatterer et al. (2014) suggested that 5 weeks of shuttle running under hypoxia attenuated the reduction in sprint time during a shuttle run test (6 $\times$ 40-m sprints with 20-s passive recovery) compared with the same training in normoxia among young male soccer players. Although these findings suggest that RSH enhances anaerobic performance, further research is required using team sport athletes (Girard et al. 2013). In addition, the efficacy of RSH has been shown in male but not female team sport players (Galvin et al. 2013; Brocherie et al. 2015a, b). Because arterial $\mathrm{O}_{2}$ desaturation has been shown to be less sensitive to hypoxic stimuli in females than in males (Billaut and Smith 2009), the effect of RSH might be small in female athletes. Therefore, this study determined the effect of 4 weeks of RSH on the repeated sprint ability of female team sport athletes.

\section{Methods}

\section{Subjects}

Thirty-two college female athletes participated in this study (Table 1). All athletes were born and living at sea level. They belonged to the lacrosse club at the same university and performed lacrosse-specific training 5 days per week ( $2 \mathrm{~h} /$ day). This study was conducted during the basic training phase in a periodized training program.

\section{Experimental design}

The experiment began with a familiarization session with sprint training (under normoxia) before the baseline measurement. The baseline measurement was then made at least $48 \mathrm{~h}$ after the familiarization session. Based on the results of repeated sprint test during the baseline measurements, all subjects were assigned randomly to either the training group in normoxia (RSN group, $\mathrm{n}=16$ ) or the training group in hypoxia ( $\mathrm{RSH}$ group, $\mathrm{n}=16$ ), matching the exercise capacity and physical characteristics (Table 1). No significant difference

Table 1 Physical characteristics and baseline measures of performance of the subjects in two groups

\begin{tabular}{lrr}
\hline & RSH group & RSN group \\
\hline Age (years) & $20 \pm 0.2$ & $20 \pm 0.1$ \\
Height (cm) & $158.9 \pm 1.4$ & $158.9 \pm 1.3$ \\
Body weight (kg) & $53.9 \pm 1.2$ & $54.6 \pm 1.6$ \\
Repeated sprint test & & \\
Peak power (W/kg) & $6.8 \pm 0.1$ & $6.9 \pm 0.1$ \\
Mean power (W/kg) & $5.6 \pm 0.1$ & $5.6 \pm 0.1$ \\
Fatigue index (\%) & $12.2 \pm 1.6$ & $13.9 \pm 1.5$ \\
\hline
\end{tabular}

Values are mean $\pm \mathrm{SE}$. between the two groups was observed at the start of the training. All training sessions were conducted in an environmentally controlled chamber using an electromagnetically braked cycle ergometer (Power Max VIII; Konami Corporation, Tokyo, Japan). The subjects performed repeated pedaling with maximal effort twice per week for 4 weeks (eight training sessions in total) under normoxic conditions $\left(\mathrm{F}_{\mathrm{i}} \mathrm{O}_{2}, 20.9 \%\right)$ or hypoxic conditions $\left(\mathrm{F}_{\mathrm{i}} \mathrm{O}_{2}\right.$, $14.5 \%$, equivalent to a simulated altitude of $3,000 \mathrm{~m}$ ). This study was conducted in a single-blind fashion, and no subject was given information about group classification. The temperature in the chamber was maintained at $20^{\circ} \mathrm{C}$ during all training sessions. The hypoxic chamber was the whole-room type, and the hypoxic condition was established by insufflation of nitrogen (Morishima et al. 2013, 2014a; Morishima and Goto 2014). The oxygen and carbon dioxide concentrations within the chamber were continuously monitored.

A standardized warm-up (30 s of submaximal sprinting and $2 \times 3$-s maximal sprinting) was performed on an ergometer. Each training session comprised two successive sets of $10 \times 7$-s sprints (maximal pedaling) with a 30-s rest period between sprints. The training protocol was designed in accordance with published protocols to enhance anaerobic performance and muscular adaptation (Edge et al. 2005; Faiss et al. 2013b). The physiological target for the training was improvements in the repeated sprint ability and buffer capacity (Edge et al. 2005; Buchheit and Laursen 2013). The load during pedaling corresponded to $4.0 \%$ of the subject's body weight. The rest period between the two sets was set at $20 \mathrm{~min}$ during the first 2 weeks, and it was shortened to $10 \mathrm{~min}$ during the subsequent 2 weeks (Figure 1). Each training session was started after a 10 -min rest following entrance into the chamber, and the subjects remained in the chamber for $10 \mathrm{~min}$ after completing the final set of training. Therefore, the total duration of exposure to hypoxia or normoxia was approximately $60 \mathrm{~min}$ in each training session. During all training sessions, the percutaneous oxygen saturation $\left(\mathrm{SpO}_{2}\right)$ was monitored using a finger pulse oximeter (Smart Pulse; Fukuda Denshi, Tokyo, Japan) placed on the tip of the right forefinger. Before and after the training period, the repeated sprint ability $(10 \times 7$-s sprints with a 30-s rest period between sprints) and maximal oxygen uptake $\left(\dot{\mathrm{VO}}_{2 \max }\right)$ were determined under normoxic conditions.

\section{Measuring maximal oxygen uptake and repeated sprint ability}

The subjects visited the laboratory twice before the start of the 4-week training program. On the first visit, the subjects' $\dot{\mathrm{V}} \mathrm{O}_{2 \mathrm{max}}$ was assessed using a graded power 


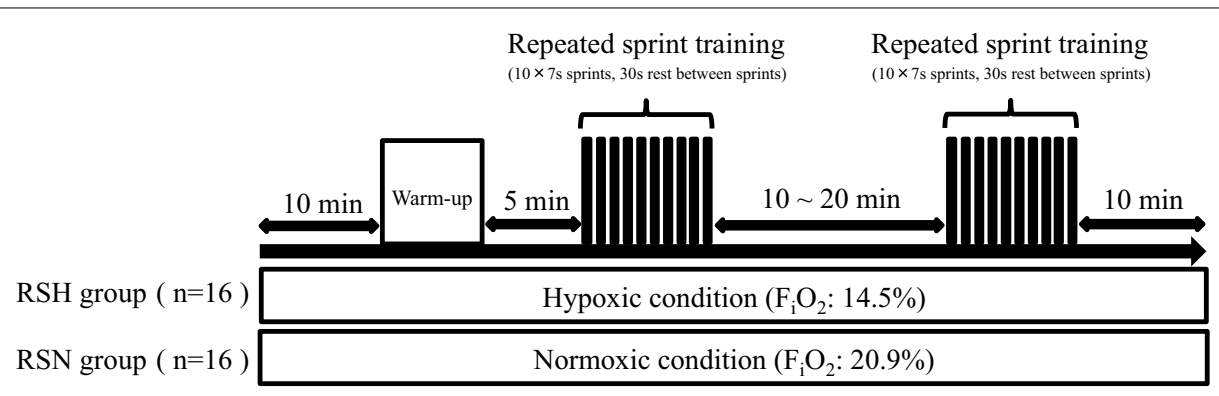

Figure 1 Protocol for each training session.

test on an ergometer (Aerobike 75XLIII; Konami Corporation, Tokyo, Japan). Before the graded power test, the subjects performed a standardized warm-up (pedaling at $50 \mathrm{~W}$ for $5 \mathrm{~min}$ ). The test began at $70 \mathrm{~W}$, and the load was increased progressively by $35-\mathrm{W}$ increments every 2 min until exhaustion (70 rpm). The TTE was also evaluated during the test. The TTE was defined as the exercise duration until the subjects failed to maintain a pedaling frequency of $70 \mathrm{rpm}$ for $5 \mathrm{~s}$. For the graded power test, the first criterion of exhaustion was maintenance of pedaling frequency. In addition to the first criterion, when the two of four criteria $\left(1 . \dot{\mathrm{V}} \mathrm{O}_{2 \mathrm{max}}\right.$ plateau, 2 . respiratory exchange ratio $\geq 1.10,3$. Heart rate (HR) reaching at least $90 \%$ of the theoretical maximal $\mathrm{HR}, 4$. rating of perceived exertion $\geq 19$ ) were fulfilled, the test was terminated (Howley et al. 1995). Respiratory gases were collected and analyzed using an automatic gas analyzer (AE300S; Minato Medical Science, Tokyo, Japan). The collected data were averaged every 30 s. Each subject's HR during exercise was measured continuously using a wireless HR monitor (RS400; Polar Electro, Tokyo, Japan). The rating of perceived exertion was determined every 2 min using a Borg 15-point scale (Borg 1973).

On the second visit, the subjects conducted a repeated sprint test under normoxic conditions, comprising $10 \times 7$-s sprints (maximal pedaling) with a 30 -s rest period between sprints. Before the tests, the subjects completed a standardized warm-up (30 s of submaximal pedaling and $2 \times 3$-s sprint) on an electromagnetically braked cycle ergometer (Power Max VIII; Konami Corporation, Tokyo, Japan). The applied load for the repeated sprint test was equivalent to $4.0 \%$ of the subject's body weight. The peak and mean power outputs during each sprint were recorded. The fatigue index was calculated as the magnitude of the percentage reduction over 10 sprints [(sprint $1-$ sprint 10)/sprint 1] $\times 100$ (Glaister et al. 2008). After the training period, a graded power test and repeated sprint test were performed to assess $\dot{\mathrm{VO}} 2 \max$.

\section{Statistical analysis}

All data are expressed as mean \pm standard error (SE). To compare power output during the repeated sprint test, two-way analysis of variance (ANOVA) with repeated measures was applied to confirm the interaction [training period (before, after training period) $\times$ number of sprints (sprints $1-10$ )] or main effect (training period, number of sprints). When ANOVA revealed a significant interaction or main effect, the Tukey-Kramer post hoc test was performed to identify differences. To compare $\dot{\mathrm{V}} \mathrm{O}_{2 \max }$ and TTE between before and after the training period, two-way ANOVA with repeated measures [group (RSH, RSN group), training period (before, after training period)] was performed. Percentage changes in the power output during the repeated sprint test (relative to pretraining values) were compared between the two groups using an unpaired $t$ test. For all tests, $P<0.05$ was considered to indicate statistical significance.

\section{Results}

Physiological and performance variables during the training period

The average values of $\mathrm{SpO}_{2}$ during all training sessions were significantly lower in the RSH group $(92.5 \pm 0.3 \%)$ than in the RSN group $(97.7 \pm 0.4 \%, P<0.05)$. Body weight did not change significantly after the training period in the RSH group (before, $53.9 \pm 1.2 \mathrm{~kg}$; after, $54.3 \pm 1.2 \mathrm{~kg}$; n.s.) or the RSN group (before, $54.6 \pm 1.6 \mathrm{~kg}$; after, $54.6 \pm 1.6 \mathrm{~kg}$; n.s.).

During the 4-week training period, both groups showed increases in power output during the training sessions, but the temporal changes differed between the groups. In the RSH group, significant increases in the peak power output (relative to the value for the first training session) were observed during training sessions 4 and $6-8(P<0.05)$, whereas the RSN group did not show a significant increase relative to the first training session over all training sessions. During sessions 3, 4 , and 6-8, the relative values of the peak power output were significantly higher in the RSH group than in the 
RSN group $(P<0.05)$. Both groups showed significant increases in the mean power output during the eight training sessions. However, the RSH group showed a significant increase in the relative value of the mean power output in session 4 , whereas the RSN group showed a significant increase in session 8 .

\section{Performance during the repeated sprint test}

Figure 2 presents the mean power output during the repeated sprint test before and after the training period. The mean power output during each sprint decreased significantly with increased numbers of sprints in both groups $(P<0.05)$. In the RSH group, significant increases (relative to the pretraining values) in the mean power output were observed in all sprints $(P<0.05)$, whereas the RSN group showed significant increases in sprints 5 and $7-10(P<0.05)$.

When the magnitude of the percentage changes (relative to pretraining values) in the power output during the repeated sprint test was compared between the two groups (Figure 3), the RSH group showed a significantly greater percentage increase in peak power output than did the RSN group $(5.0 \pm 0.7 \%$ vs. $1.5 \pm 0.9 \%$, respectively; $P<0.05$ ). In addition, the RSH group showed a significantly greater percentage increase in the average values of mean power output over 10 sprints than did the RSN group $(9.7 \pm 0.9 \%$ vs. $6.0 \pm 0.8 \%$, respectively; $P<0.05)$. When the fatigue index during the repeated sprint test was compared between before and after the training period, the RSN group showed a significant reduction in the fatigue index after the training period (before, $13.9 \pm 1.5 \%$; after, $9.6 \pm 1.1 \% ; P<0.05$ ). The RSH group also showed a tendency toward a reduction after the training period (before, $12.2 \pm 1.6 \%$; after, $9.2 \pm 0.9 \%$; $P=0.05)$.

\section{$\dot{\mathrm{V}}_{2 \mathrm{max}}$ and time to exhaustion}

There were no significant differences in $\dot{\mathrm{V}} \mathrm{O}_{2}$ max or maximal HR between the two groups before the training period (Table 2). These values were not significantly different in either group between before and after the training period. However, the RSH group showed a significant increase in TTE after the training period $(P<0.05)$, whereas no significant change was observed in the RSN group. The maximal HR did not change significantly after the training period in either group.

\section{Discussion}

This study compared the effects of 4 weeks of repeated sprint training between a training group in hypoxia (RSH group) and a training group in normoxia (RSN group). The results indicated that the RSH group showed significantly greater improvement in repeated sprint ability than did the RSN group. A novel finding of this study was that an approximately three-fold greater increase in peak power output during repeated sprint test was observed in the RSH group than in the RSN group. To our knowledge, this is the first report to show that high-intensity training under hypoxic conditions is beneficial for improving maximal power output.

Both groups showed significant increases in the mean power output during the repeated sprint test after the 4-week training period. However, when we compared the relative increase from the pretraining values between the
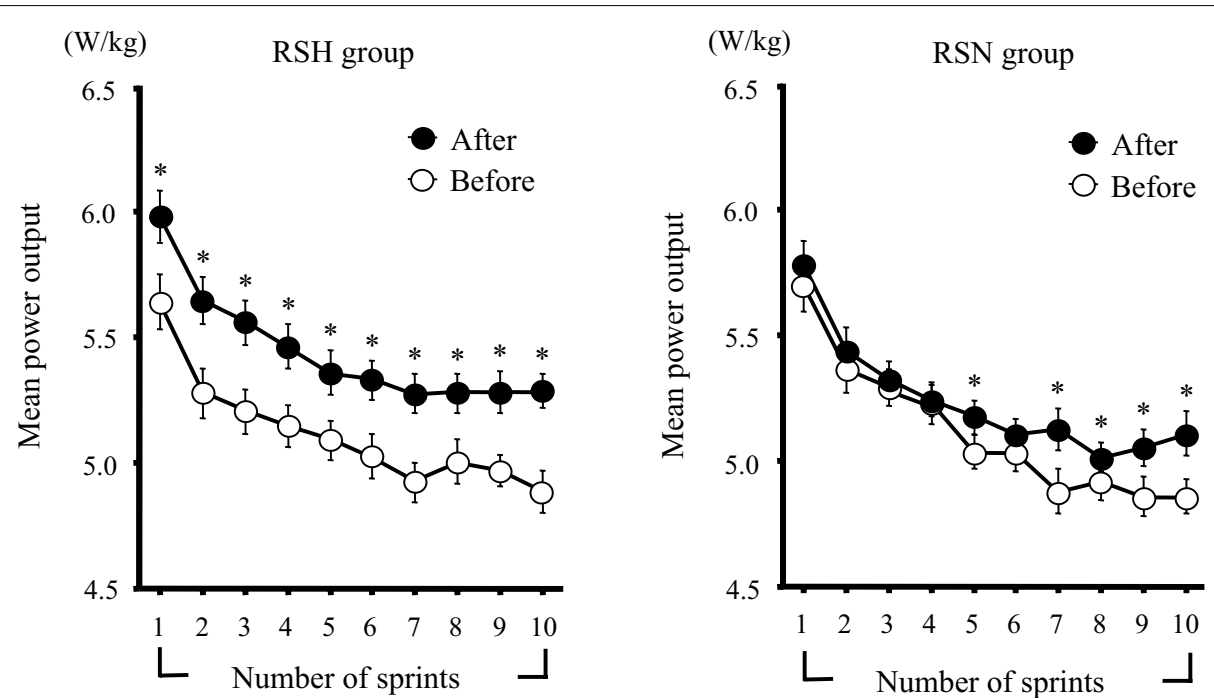

Figure 2 Time-course changes in the mean power output during the repeated sprint test in the RSH and RSN groups before and after the training period. Values are mean $\pm \mathrm{SE} .{ }^{*} P<0.05 \mathrm{vs}$. corresponding value before the training period. 

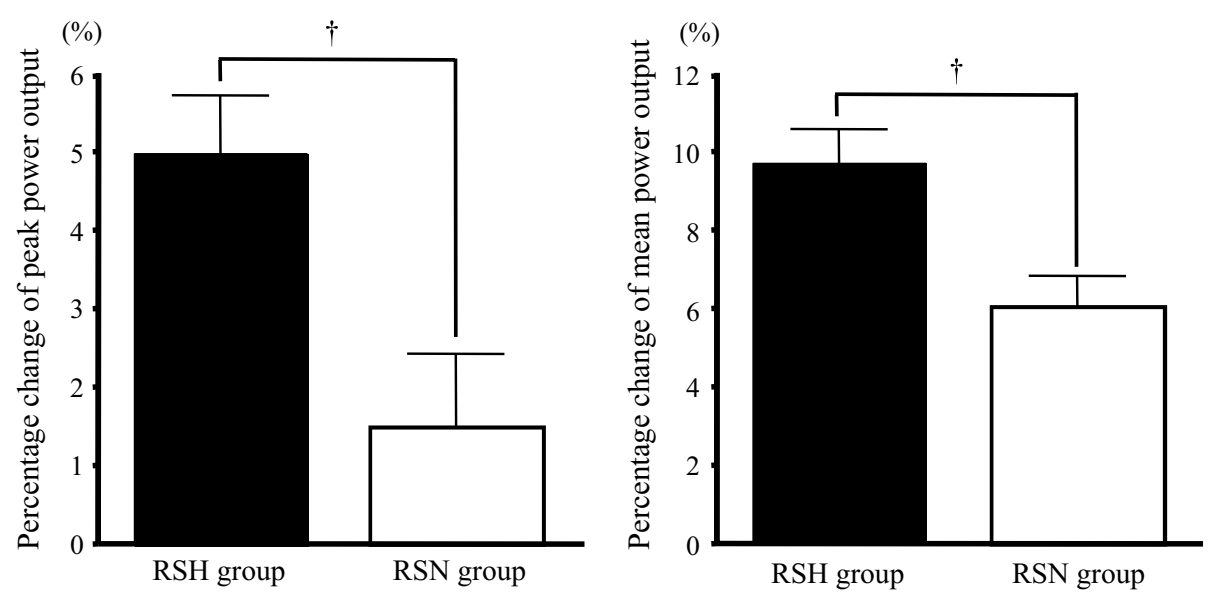

Figure 3 Percentage changes in the peak and mean power outputs during the repeated sprint test before and after the training period. Values are mean \pm SE. ${ }^{\dagger} P<0.05$ between the RSH and RSN groups.

Table 2 Respiratory variables and performance during the graded power test

\begin{tabular}{llc}
\hline & Before & After \\
\hline$\dot{\mathrm{V}} \mathrm{O}_{2 \text { max }}(\mathrm{ml} / \mathrm{min})$ & & \\
$\mathrm{RSH}$ group & $2,398 \pm 52$ & $2,470 \pm 59$ \\
$\mathrm{RSN}$ group & $2,461 \pm 79$ & $2,473 \pm 67$ \\
$\dot{\mathrm{V}} \mathrm{O}_{2 \mathrm{max}} / \mathrm{BW}(\mathrm{ml} / \mathrm{min} / \mathrm{kg})$ & & \\
$\mathrm{RSH}$ group & $44.6 \pm 0.8$ & $45.9 \pm 0.7$ \\
RSN group & $45.1 \pm 1.0$ & $45.4 \pm 0.8$ \\
Time to exhaustion (s) & & $688 \pm 21^{*}$ \\
RSH group & $659 \pm 18$ & $678 \pm 22$ \\
RSN group & $656 \pm 23$ & $193 \pm 1$ \\
Maximal HR (bpm) & & $189 \pm 2$ \\
RSH group & $194 \pm 2$ & \\
RSN group & $190 \pm 2$ & \\
\hline
\end{tabular}

Values are mean $\pm \mathrm{SE}$.

${ }^{*} \mathrm{P}<0.05$ between before and after the training period.

two groups, the RSH group showed a significantly greater percentage change than did the RSN group. This result suggests that the RSH group had further improvement in the repeated sprint ability compared with the RSN group. These findings are supported by recent publications. Hamlin et al. (2010) reported that sprint training in moderate hypoxia $\left(\mathrm{F}_{\mathrm{i}} \mathrm{O}_{2}, 17.0-14.0 \%\right)$ for 10 successive days caused significantly greater increases in the mean power output during $30 \mathrm{~s}$ of maximal pedaling than the same training in normoxia. Faiss et al. (2013a) also showed that 4 weeks of RSH ( 3 sets of $5 \times 10$-s sprint; $\mathrm{F}_{\mathrm{i}} \mathrm{O}_{2}, 14.6 \%$ ) resulted in a significant increase in the number of sets until exhaustion during the repeated sprint test, whereas no change was found after the same training in normoxia.
Galvin et al. (2013) recently reported that 4 weeks of RSH $\left(\mathrm{F}_{\mathrm{i}} \mathrm{O}_{2}, 13.0 \%\right)$ resulted in two-fold greater improvements in distance during the intermittent running test than did equivalent RSN. The repeated sprint ability is related to the capacity for phosphocreatine (PCr) resynthesis (Mendez-Villanueva et al. 2012) and aerobic capacity (Edge et al. 2005; Bishop et al. 2011). Although we were unable to evaluate the capacity for $\mathrm{PCr}$ resynthesis, $\mathrm{VO}_{2 m a x}$ (an indicator of aerobic capacity) did not change significantly in either group after the training period. During shortduration, high-intensity exercise of $<60 \mathrm{~s}$, the metabolic response under hypoxia has been suggested to differ from that under normoxia, independent of similar exercise capacities (Weyand et al. 1999). Two studies (Ogura et al. 2006; Ogawa et al. 2007) indicated that the power output during $40 \mathrm{~s}$ of maximal pedaling or a maximal anaerobic running test did not differ between hypoxic and normoxic conditions. However, the contribution of the anaerobic energy supply was augmented by about 9.3\% in hypoxia compared with normoxia (Ogura et al. 2006). The augmented energy supply from the anaerobic system might cause greater stimulus for adaptation and further improvement in the repeated sprint ability.

The novel finding of this study was that the power output during the first sprint in the repeated sprint test increased significantly only in the RSH group. In the repeated sprint test, a significant increase in power output during all sprints was observed in the RSH group, whereas the RSN group showed significant increases during the second half of the repeated sprint exercise. However, growing evidence suggests that RSH and highintensity interval training under hypoxic conditions produce greater improvements in the mean power output (anaerobic endurance capacity) (Hamlin et al. 2010; Faiss 
et al. 2013a, b; Galvin et al. 2013). Our finding of further improvement in the power output of the first sprint has not been reported. During 7-s maximal pedaling, the ATP-PCr system rather than the glycolytic system is thought to play a main role in ATP production. Therefore, the improved power output during the first sprint in the RSH group might be related to an augmented $\mathrm{PCr}$ content in muscle. In support of this hypothesis, we recently observed that six consecutive days of RSH significantly increased the $\mathrm{PCr}$ content in muscle among sprinters (unpublished observation). Further research involving determination of the intramuscular $\mathrm{PCr}$ content is necessary.

In this study, we monitored the time course of the changes in power output during all training sessions in each group. Consequently, the RSH group showed a significantly greater increase in power output during the second week (versus the value in first training session), whereas a similar increase was observed during fourth week in the RSN group. Therefore, the training stimulus might be higher in the RSH group during the training period, leading to greater adaptation of sprint performance. Furthermore, the fatigue index was reduced significantly after the training period in the RSN group alone. However, caution is necessary when interpreting this later result because the absence of significant improvement in the RSH group is thought to be due to marked enhancement of the power output in the first sprint during repeated sprint test.

A unique point of this study is that we recruited female team sport athletes. Although we found marked improvements in repeated sprint ability, we cannot conclude whether the training effects in this study are specific to female athletes because of a lack of comparative data from males. Because female athletes tend to have lower anaerobic power outputs than do male athletes (Brooks et al. 1990; Billaut and Smith 2009), there might be greater trainability in response to sprint training. In contrast, females have been shown to be less sensitive to hypoxic stimuli for arterial $\mathrm{O}_{2}$ desaturation (Billaut and Smith 2009). Therefore, it is unlikely that our results are specific to female athletes. We believe that RSH is suitable for both male and female athletes.

In this study, twice-weekly repeated sprint training sessions (approximately $60 \mathrm{~min}$ per training session) were incorporated into the regular training schedule. Therefore, the training protocol is practical and would be suitable for most athletes. Our finding suggests that RSH is a new strategy for augmenting the maximal anaerobic power output and repeated sprint ability in female athletes. This study focused on the adaptation of sprint performance, and future studies are necessary to reveal the detailed mechanism behind the adaptation.

\section{Conclusion}

Four weeks of RSH caused further increases in the peak and mean power outputs during the repeated sprint test compared with RSN in female team sport athletes.

\section{Authors' contributions}

NK and KG participated in the critical conception and design, the acquisition of data, analysis and interpretation of data, drafting of the manuscript, and critical revision of the manuscript, and approved the final version. SM, SI, ES, MM participated for data collection and interpretation of data. All authors read and approved the final manuscript.

\section{Author details}

${ }^{1}$ Graduate School of Sport and Health Science, Ritsumeikan University, Kusatsu, Shiga, Japan. ${ }^{2}$ Faculty of Sport and Health Science, Ritsumeikan University, 1-1-1 Nojihigashi, Kusatsu, Shiga 525-8577, Japan.

\section{Acknowledgments}

The authors thank all subjects who conducted the intensive training. The authors acknowledge Takuma Morishima, Yuta Hasegawa, Toshiaki ljichi, Hiroto Sasaki, and Ayaka Mori for the excellent technical support during the experiment. This study was supported by a research grant from the Japan Society for the Promotion of Science.

\section{Funding}

Japan Society for the Promotion of Science.

Compliance with ethical guidelines

Competing interests

The authors declare that they have no competing interests.

\section{Consent for publication}

This study was approved by the Ethics Committee for Human Experiments at Ritsumeikan University, Japan.

Received: 28 January 2015 Accepted: 13 May 2015

Published online: 02 July 2015

\section{References}

Billaut F, Smith K (2009) Sex alters impact of repeated bouts of sprint exercise on neuromuscular activity in trained athletes. Appl Physiol Nutr Metab 34:689-699

Billaut F, Gore CJ, Aughey RJ (2012) Enhancing team-sport athlete performance: is altitude training relevant? Sports Med 42:751-767

Bishop D, Edge J (2006) Determinants of repeated-sprint ability in females matched for single-sprint performance. Eur J Appl Physiol 97:373-379

Bishop D, Girard O, Mendez-Villanueva A (2011) Repeated-sprint ability_part II: recommendations for training. Sports Med 41:741-756

Borg GA (1973) Perceived exertion: a note on "history" and methods. Med Sci Sports 5:90-93

Brocherie F, Girard O, Faiss R, Millet GP (2015a) High-intensity intermittent training in hypoxia: a double-blinded, placebo-controlled field study in youth football players. J Strength Cond Res 29:226-237

Brocherie F, Millet GP, Hauser A, Steiner T, Rysman J, Wehrlin JP et al (2015b) "Live high-train low and high" hypoxic training improves team-sport performance. Med Sci Sports Exerc. doi:10.1249/MSS.0000000000000630

Brooks S, Nevill ME, Meleagros L, Lakomy HK, Hall GM, Bloom SR et al (1990) The hormonal responses to repetitive brief maximal exercise in humans. Eur J Appl Physiol Occup Physiol 60:144-148

Buchheit M, Laursen PB (2013) High-intensity interval training, solutions to the programming puzzle: part I: cardiopulmonary emphasis. Sports Med 43:313-338

Dufour SP, Ponsot E, Zoll J, Doutreleau S, Lonsdorfer-Wolf E, Geny B et al (2006) Exercise training in normobaric hypoxia in endurance runners. 
I. Improvement in aerobic performance capacity. J Appl Physiol 100:1238-1248

Edge J, Bishop D, Goodman C, Dawson B (2005) Effects of high- and moderate-intensity training on metabolism and repeated sprints. Med Sci Sports Exerc 37:1975-1982

Faiss R, Leger B, Vesin JM, Fournier PE, Eggel Y, Deriaz O et al (2013a) Significant molecular and systemic adaptations after repeated sprint training in hypoxia. PLoS One 8:e56522

Faiss R, Girard O, Millet GP (2013b) Advancing hypoxic training in team sports: from intermittent hypoxic training to repeated sprint training in hypoxia. Br J Sports Med 47(Suppl 1):i45-i50

Faiss R, Willis S, Born DP, Sperlich B, Vesin JM, Holmberg HC et al (2015) Repeated double-poling sprint training in hypoxia by competitive crosscountry skiers. Med Sci Sports Exerc 47:809-817

Galvin HM, Cooke K, Sumners DP, Mileva KN, Bowtell JL (2013) Repeated sprint training in normobaric hypoxia. Br I Sports Med 47(Suppl 1):i74-i79

Gatterer H, Philippe M, Menz V, Mosbach F, Faulhaber M, Burtscher M (2014) Shuttle-run sprint training in hypoxia for youth elite soccer players: a pilot study. J Sports Sci Med 13:731-735

Girard O, Amann M, Aughey R, Billaut F, Bishop DJ, Bourdon P et al (2013) Position statement-altitude training for improving team-sport players' performance: current knowledge and unresolved issues. Br I Sports Med 47(Suppl 1):i8-i16

Glaister M, Howatson G, Pattison JR, McInnes G (2008) The reliability and validity of fatigue measures during multiple-sprint work: an issue revisited. J Strength Cond Res 22:1597-1601

Hamlin MJ, Marshall HC, Hellemans J, Ainslie PN, Anglem N (2010) Effect of intermittent hypoxic training on $20 \mathrm{~km}$ time trial and $30 \mathrm{~s}$ anaerobic performance. Scand I Med Sci Sports 20:651-661

Howley ET, Bassett DR Jr, Welch HG (1995) Criteria for maximal oxygen uptake: review and commentary. Med Sci Sports Exerc 27:1292-1301
Mendez-Villanueva A, Edge J, Suriano R, Hamer P, Bishop D (2012) The recovery of repeated-sprint exercise is associated with $\mathrm{PCr}$ resynthesis, while muscle pH and EMG amplitude remain depressed. PLoS One 7:e51977

Millet GP, Roels B, Schmitt L, Woorons X, Richalet JP (2010) Combining hypoxic methods for peak performance. Sports Med 40:1-25

Millet GP, Faiss R, Brocherie F, Girard O (2013) Hypoxic training and team sports: a challenge to traditional methods? Br J Sports Med 1:i6-i7

Mizuno M, Juel C, Bro-Rasmussen T, Mygind E, Schibye B, Rasmussen B et al (1990) Limb skeletal muscle adaptation in athletes after training at altitude. J Appl Physiol 68:496-502

Morishima T, Goto K (2014) Successive exposure to moderate hypoxia does not affect glucose metabolism and substrate oxidation in young healthy men. Springerplus 3:370

Morishima T, Kurihara T, Hamaoka T, Goto K (2013) Whole body, regional fat accumulation, and appetite-related hormonal response after hypoxic training. Clin Physiol Funct Imaging 34:90-97

Morishima T, Mori A, Sasaki H, Goto K (2014) Impact of exercise and moderate hypoxia on glycemic regulation and substrate oxidation pattern. PLoS One 9:e108629

Ogawa T, Hayashi K, Ichinose M, Wada H, Nishiyasu T (2007) Metabolic response during intermittent graded sprint running in moderate hypobaric hypoxia in competitive middle-distance runners. Eur J Appl Physiol 99:39-46

Ogura Y, Katamoto S, Uchimaru J, Takahashi K, Naito H (2006) Effects of low and high levels of moderate hypoxia on anaerobic energy release during supramaximal cycle exercise. Eur J Appl Physiol 98:41-47

Strzala M, Ostrowski A, Szygula Z (2011) Altitude training and its influence on physical endurance in swimmers. J Hum Kinet 28:91-105

Weyand PG, Lee CS, Martinez-Ruiz R, Bundle MW, Bellizzi MJ, Wright S (1999) High-speed running performance is largely unaffected by hypoxic reductions in aerobic power. J Appl Physiol 86:2059-2064

\section{Submit your manuscript to a SpringerOpen ${ }^{\circ}$ journal and benefit from:}

- Convenient online submission

- Rigorous peer review

- Immediate publication on acceptance

- Open access: articles freely available online

- High visibility within the field

- Retaining the copyright to your article

Submit your next manuscript at $\boldsymbol{\wedge}$ springeropen.com 